\title{
Specific characteristics of hemorrhagic Meckel's diverticulum at double-balloon endoscopy
}

\section{(ㅇ)( $\odot$}

\author{
Authors \\ Yasuyuki Mizutani ${ }^{1}$, Masanao Nakamura' ${ }^{1}$ Osamu Watanabe ${ }^{1}$, \\ Takeshi Yamamura², Kohei Funasaka², Eizaburo Ohno ${ }^{1}$, Hiroki \\ Kawashima ${ }^{1}$, Ryoji Miyahara' ${ }^{1}$ Alberto Murino ${ }^{3}$, Hidemi Goto ${ }^{1}$, \\ Yoshiki Hirooka ${ }^{2}$ \\ Institutions \\ 1 Department of Gastroenterology and Hepatology, Nagoya \\ University Graduate School of Medicine, Nagoya, Japan \\ Japan \\ 3 Royal Free Unit for Endoscopy, The Royal Free Hospital and \\ health, London, United Kingdom \\ submitted 24.4.2016 \\ accepted after revision $\quad 4.10 .2016$ \\ Bibliography \\ DOI http://dx.doi.org/10.1055/s-0042-119810 | \\ Endoscopy International Open 2017; 05: E35-E40 \\ (c) Georg Thieme Verlag KG Stuttgart · New York \\ ISSN 2364-3722
}

2 Department of Endoscopy, Nagoya University Hospital, Nagoya, University College London Institute for Liver and Digestive

\author{
Corresponding author \\ Masanao Nakamura, Department of Gastroenterology, Nagoya \\ University Graduate School of Medicine, 65 Tsuruma-cho, Showa- \\ ku, Nagoya 466-8550, Japan \\ Fax: 81-52-744-2180 \\ makamura@med.nagoya-u.ac.jp
}

\section{ABSTRACT}

Background and study aims Diagnosis of Meckel's diverticulum (MD) before surgery may be challenging; double-balloon endoscopy (DBE) facilitates identification of MD in the setting of a gastrointestinal bleeding; however, MD can be found incidentally without this condition. The purpose of this research was to determine specific characteristic of hemorrhagic MD and incidental MD at DBE. Patients and methods Ectopic gastric mucosa enclosed in the MD and/or ulceration were defined as "major findings"; ring-like scar surrounding the MD was defined as "minor finding". We retrospectively reviewed the medical records of patients affected by MD and analyzed the findings that significantly affected the characterization of MD.

Results MD was diagnosed in 33 patients. The axis of the diverticulum was longer in hemorrhagic MD compared to incidental MD $(P=0.031)$. The amount of transfusion was significantly higher $(P=$ 0.018 ) in the hemorrhagic MD group. Hemorrhagic MD was significantly more correlated with major findings $(P=0.01)$ and minor findings $(P<0.01)$. The specificity of major finding was $100 \%$ while the sensitivity of major and/or minor findings was $96 \%$.

Conclusions The combination of major and minor findings appears to improve the diagnostic ability of hemorrhagic MD avoiding unnecessary diverticulectomy.

\section{Introduction}

Meckel diverticulum (MD) is a congenital intestinal blind pouch that results from incomplete obliteration of the vitelline duct during the fifth week of gestation. The diverticulum is located on the antimesenteric border of the ileum, about $40 \mathrm{~cm}$ to $130 \mathrm{~cm}$ proximal to the ileocecal valve and it is usually $3 \mathrm{~cm}$ to $5 \mathrm{~cm}$ long [1]. Individuals with MD have a $4 \%$ to $6 \%$ lifetime risk of developing a complication [2]. The most common clinical presentation is gastrointestinal bleeding, which occurs in $25 \%$ to $50 \%$ of patients [3].

In case of obscure gastrointestinal bleeding with negative esophagogastroduodenoscopy (EGD) and colonoscopy, investigation of the small bowel with capsule endoscopy (SBCE) and/ or double-balloon endoscopy (DBE) is recommended [4]. In that scenario, when MD is identified it is commonly ascribed as the cause of bleeding although at times it may be just an incidental finding.
Only a few reports regarding endoscopic diagnosis of MD have been published so far. Yamamoto et al. reported the first case of MD identified during DBE [5]. Mylonaki et al. observed MD at SBCE and defined it as a "black hole with blood-filled appearance" [6]. Shinozaki et al. reported 5 cases of MD [7]: 3 were associated with ulceration, 1 with diverticulitis and in the remaining case gastrointestinal hemorrhage was caused by hemorrhagic tumor in the jejunum while the MD was only an incidental finding. The authors concluded that ulceration is the only feature related to hemorrhagic MD [7]. However, an ulcerated MD was found only in 35 of 43 pediatric patients (81\%) with gastrointestinal bleeding [8]. Therefore, the correlation between ulceration and hemorrhagic MD still remains unclear.

The acids secreted by the ectopic gastric mucosa located in the diverticulum are likely to be behind the ulceration of contiguous SB mucosal; it exposes the submucosal vessels and triggers the gastrointestinal bleeding [8-10]. The healing process that occurs afterwards, promotes a submucosal fibrosis around 

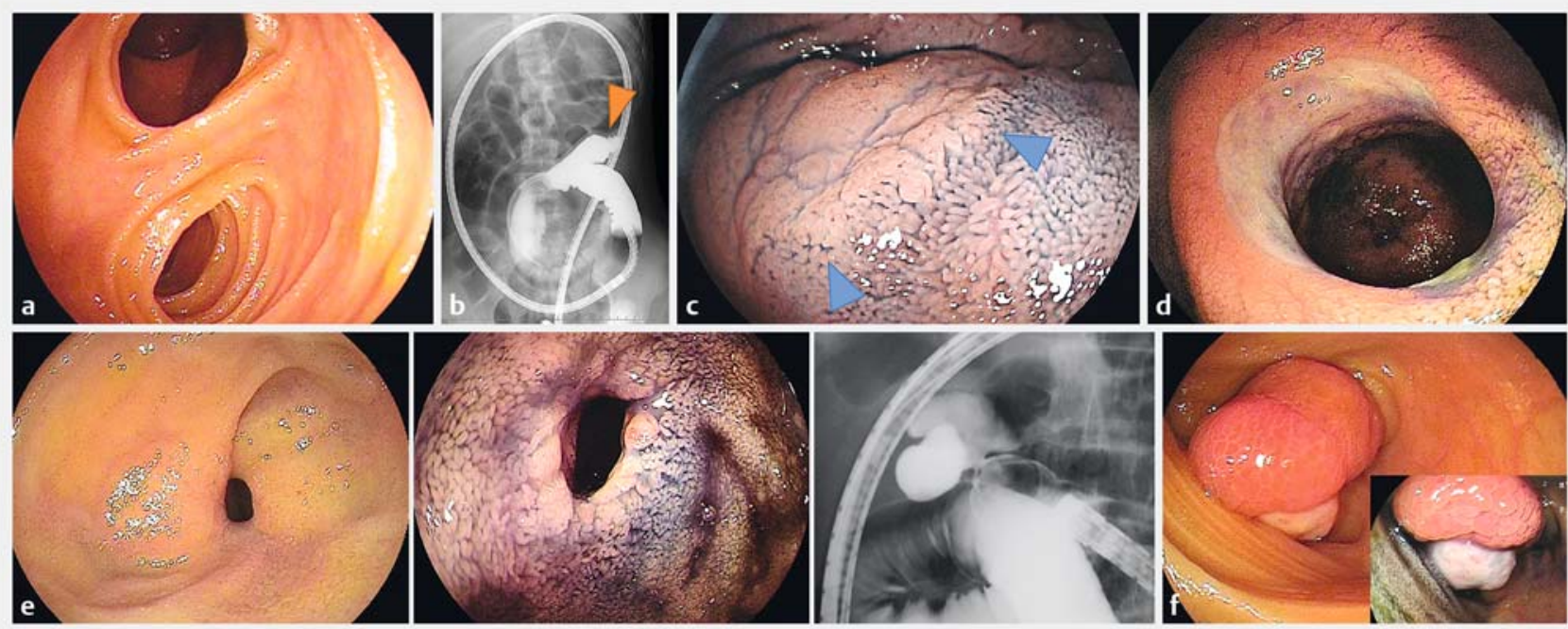

Fig. 1 a MD was defined as a double lumen sign within $100 \mathrm{~cm}$ proximally to the ileo-caecal valve at DBE. $\mathbf{b}$ Location of MD as antimesenteric side of the middle-to-distal ileum by $\mathrm{x}$-ray examination injecting gastrografin through the enteroscope working channel. c Ectopic gastric mucosa emphasized by indigo carmine dye. Blue arrowheads indicate ectopic mucosa. $\mathbf{d}$ Meckel's diverticulum ulceration. e ring like scar (left). Scar emphasized by indigo carmine (center). MD fluoroscopic appearance (right). f Inverted and ulcerated Meckel's diverticulum.

the MD, which acquires the shape of a ring-like scar stricture. Thus, the ectopic gastric mucosa and the ulceration seem to be the primary cause of the gastrointestinal bleeding, the ringlike scar indirectly indicates the existence of heterotopic mucosa.

The primary aim of this study was to reveal the clinical and endoscopic features of hemorrhagic and incidental MD. The secondary aim was to relate these findings with the diagnostic accuracy of hemorrhagic MD.

\section{Patients and methods}

\section{Patients}

This was a retrospective study performed at the Nagoya University Hospital. Data regarding 1747 patients who underwent 1991 DBEs between January 2003 and December 2015 were reviewed. Patients with MD were selected. In addition, the surgical specimens and the histopathologic reports of the heterotopic mucosa of the MD were reviewed.

Diagnosis of MD was made when a double lumen was identified within $100 \mathrm{~cm}$ proximal to the ileocecal valve ( $\mathbf{F i g . 1 a ) . ~}$ The diagnosed of MD was confirmed afterwards by assessing the antimesenteric surface of the middle-to-distal ileum with fluoroscopy during DBE ( $\mathbf{F i g}$. 1b) or with surgery.

MD was defined as hemorrhagic or incidental. Diagnosis of hemorrhagic MD was associated with gastrointestinal bleeding (detected on contrast computed tomography (CT) and/or on angiography) and lack of gastrointestinal bleeding after surgical diverticulectomy. Incidental MD was an incidental finding without gastrointestinal bleeding. Biopsies were taken from incidental MD to confirm the absence of ectopic gastric mucosa.

Indigo carmine was used during DBE when a MD was en-



When the endoscopic assessment of MD was not feasible due to a tight orifice - fluoroscopy was then performed ( $\triangleright$ Fig. 1 e).

Ectopic gastric mucosa and ulceration ( $>$ Fig. 1c, $>$ Fig. 1d) were defined as "major findings" while the ring-like scar appearance ( $\triangleright$ Fig. 1e) was a "minor finding" (because it indirectly suggests the existence of heterotopic mucosa).

\section{Analysis}

We compared the clinical features and endoscopic findings of hemorrhagic and incidental MD. Primary endpoints were sensitivity and specificity of major and minor findings at DBE for diagnosis of hemorrhagic MD. Secondary endpoints were clinical features of hemorrhagic MD patients.

\section{Statistical analysis}

SPSS version 22 for Windows was used for data analysis. The results are presented as mean, mean \pm standard deviation, range, percentage, or median. Differences in the categorical variables were examined using the Chi-square test or Fisher's exact probability test and odds ratios and $95 \%$ confidence intervals $(\mathrm{Cl})$ were used to quantify major and minor findings. Mean values were compared by Mann-Whitney U-test. $P$ values $<0.05$ were considered statistically significant.

\section{Results}

MD was diagnosed in 33 patients ( $>$ Table 1 ). Mean age was $33.0 \pm 20.6$ years ( $9-78$ years). A total of 29 patients presented with bloody stools and/or anemia while 4 patients suffered from abdominal pain. Symptom duration was 21 days (range 2 -4675). Twenty-nine patients had retrograde DBE, 1 patient had antegrade DBE, and 3 patients underwent both antero- 
- Table 1 Characteristics of 33 patients with MD.

\begin{tabular}{|l|c|}
\hline Features & $\mathbf{n}$ \\
\hline Number of patients & 33 \\
\hline Age,mean \pm SD, year & $33.0 \pm 20.6$ \\
\hline Sex, male/female, $n$ & $24 / 9$ \\
\hline Complaints & \\
\hline facal occult blood & 4 \\
\hline ileus symptom & 4 \\
\hline iron deficient anemia + Melena/hematochezia & 25 \\
\hline Duration of symptoms, days, median (range) & $21(2-4675)$ \\
\hline Approaches & \\
\hline Antegrade & 1 \\
\hline Retrograde & 29 \\
\hline Antegrade + Retrograde & 3 \\
\hline Leision in MD (the mean from ileocecal valve), cm & $56.2 \pm 9.9$ \\
\hline Pathology & \\
\hline Hemorrhagic MD ( $n=25$ ) & 18 \\
\hline ectopic gastric mucosa & 1 \\
\hline heterotopic pancreatic tissue & 6 \\
\hline only mucosa of small intestine & \\
\hline Intussusception caused by MD ( $n=3$ ) & \\
\hline only mucosa of small intestine & \\
\hline Observation ( $n=5$ ) & \\
\hline only mucosa of small intestine(biopsy) & \\
\hline
\end{tabular}

grade and retrograde DBE. All MDs were identified using the retrograde DBE procedure. Only 1 incidental MD was seen during a anterograde DBE performed in a patient who had undergone pancreaticoduodenectomy. Twenty-five patients diagnosed with hemorrhagic MD presented with hematochzia and iron deficiency anemia, which did not recur for more than 1 year after the diverticulotomy. Three patients with incidental MD presented with ileus because of inverted MD while 4 patients had hematochzia and iron deficiency anemia and 1 patient had abdominal pain ( $\mathbf{F i g . 2}$ ). The indications to perform DBE in case of incidental MD were 3 cases of ileus, 2 cases of nonsteroidal anti-inflammatory drugs (NSAIDs) enteritis, anastomotic ulceration, gynecological disorders, and pseudo ileus ( Fig.2).

We adopted a wait-and-see approach for patients who were diagnosed with incidental MD; however, they either did not experience a recurrence of bleeding or experienced bleeding because of the primary disease not related with the MD.

One patient with ulceration and a ring-like scar was diagnosed with NSAID-induced small bowel disorder rather than hemorrhagic MD because multiple ulcerations were seen throughout the small bowel and the patient's symptoms resolved after NSAID cessation.

On average, MD was identified at $56.2 \pm 9.9 \mathrm{~cm}$ from the ileocecal valve. All patients with gastroinestinal bleeding and incidental MD had previously undergone EGD and colonoscopy and/or contrast-enhanced CT. SBCE was performed before DBE in 14 patients, and 6 of them showed MD (43\%). All patients who underwent SBCE had a ring-like scar. Meckel's scan was performed for 23 patients and MD was detected in 8 (35\%).

As for the results of the surgical specimen examination of the 25 hemorrhagic MDs, 18 specimens had ectopic gastric mucosa, 1 specimen had heterotopic pancreatic tissue while small bowel mucosa was determined in 6 specimens. All patients who underwent diverticulectomy had 1-year follow-up without developing anemia.

\section{Comparison of endoscopic findings of hemorrhagic MD and incidental MD}

A significant difference between detection of hemorrhagic MD and incidental MD was observed (OR 2.1; $95 \% \mathrm{Cl} 1.39-3.13$; $P$ $=0.01)$ ( $\downarrow$ Table 2$)$. However, the detection rate for ectopic gastric mucosa and open ulcer among the 2 MD groups was not significant $(P=0.31$ and $P=0.16$, respectively). The detection rate of the minor finding in the hemorrhagic MD group was significantly higher than that in the incidental MD group (OR 51.3; $95 \% \mathrm{Cl} 4.58-576.0 ; P<0.01)$. In the hemorrhagic MD group, only 1 patient did not have any major or minor findings and treatment was difficult in this case because of excessive clotting. In the incidental MD group, only 1 patient had a minor finding, having a ring-like scar caused by NSAID-induced enteritis ( Table 3 ).

Inverted MD was seen in 5 patients. Two of them had inverted hemorrhagic MD with major or minor findings. One was ulcerated ( $\triangleright$ Fig. 1f) and the other one presented with a scar. The other 3 patients had ileus because of intussusception of inverted MD.

Diagnostic accuracy for hemorrhagic MD based on major and minor findings at DBE is shown in $>$ Table 4 . Specificity of the major findings was $100 \%$, sensitivity of the major and/or minor findings was $96 \%$, and the accuracy was $94 \%$ ( $>$ Table 4).

\section{Comparison of the clinical features of hemorrhagic MD and incidental MD}

The median ages were 24 years (range $9-77$ ) and 44 years (range 21-78) for patients with hemorrhagic and incidental MD, respectively $(P=0.057)$. Although there was no significant difference, hemorrhagic MD was found mostly in younger patients. The duration of symptoms in hemorrhagic MD was 358 \pm 968 days, which was longer compared with incidental MD, $122 \pm 172$ days. The median of the major axis of hemorrhagic MD on fluoroscopy at DBE was $6 \mathrm{~cm}(4-12)$, and it was significantly longer than that of incidental MD. which measured $4 \mathrm{~cm}$ $(2-6)(P=0.031)$. Moreover, patients with hemorrhagic MD often developed massive melena requiring 5.2 units $(0-55)$, while patients with incidental MD did not require any transfusion $(P=0.018)$. Of the 13 patients with hemorrhagic MD who underwent SBCE, MD was detected in 6 patients $(46.1 \%)$ while 


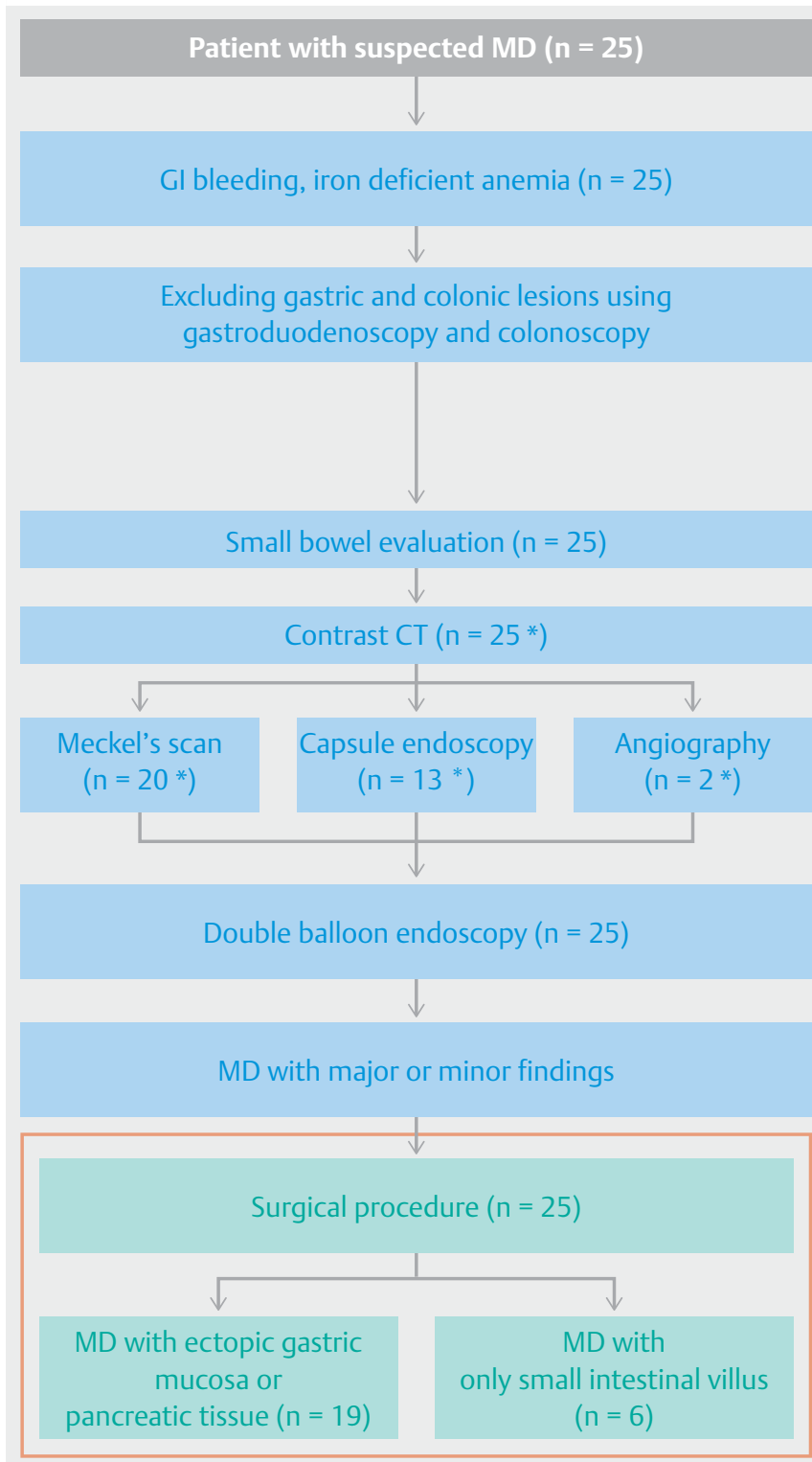

Hemorrhagic MD $(n=25)$

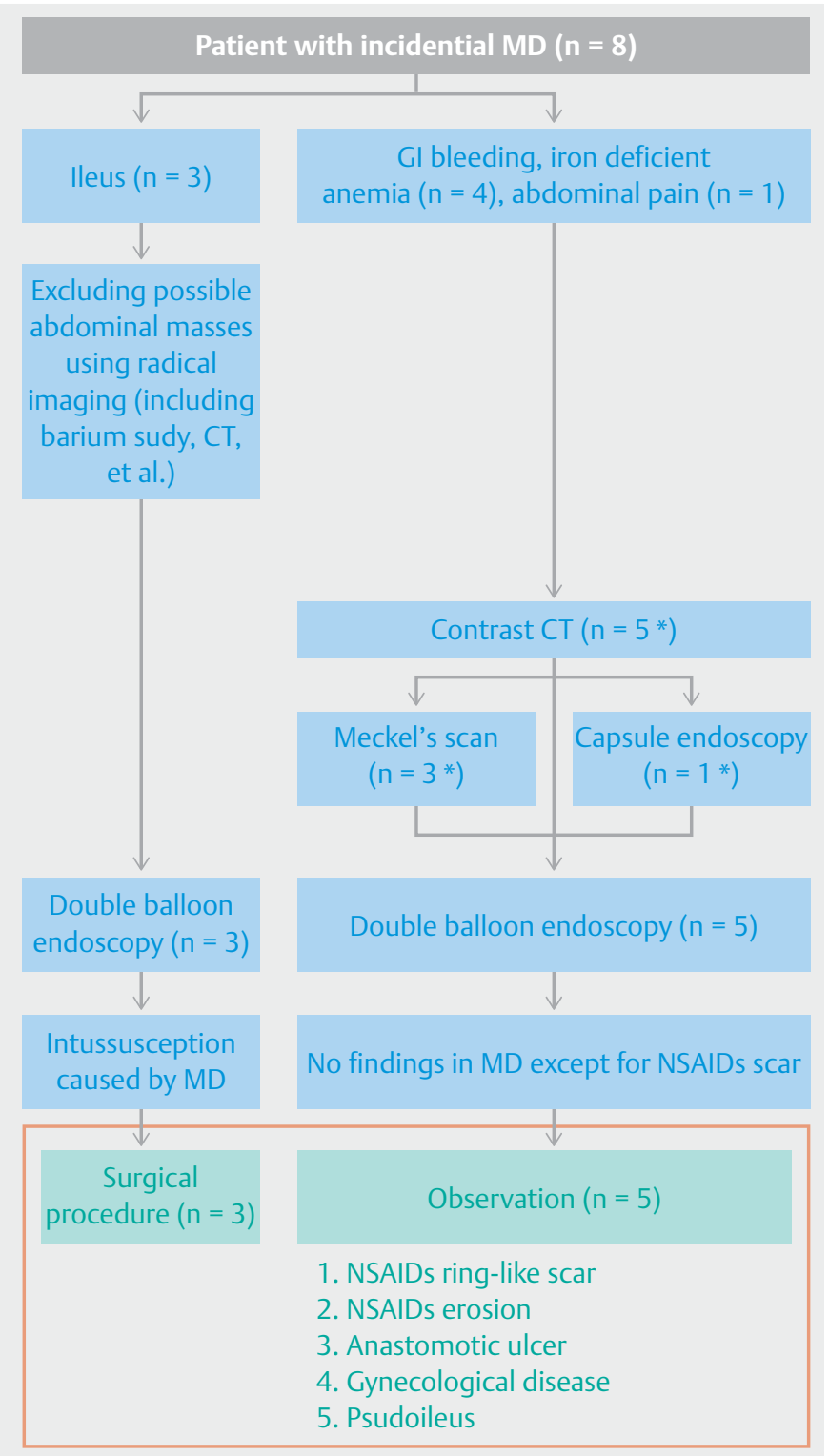

Incidental MD ( $\mathrm{n}=8)$

- Fig. 2 Endoscopic and therapeutic managements of patients with MD. * There is duplication.

the only SBCE performed in incidental MD did not identify it $(0 \%)$. Of the 20 patients with hemorrhagic MD who underwent the Meckel scan, a positive result was obtained in 8 cases (40\%). The MD did not have ectopic mucosa in 1 of these 8 patients. In patients with incidental MD, the Meckel scan was performed in 3 cases, and MD was not detected. Comparison between SBCE and Meckel Scan did not show a significant difference.

\section{Discussion}

DBE is generally considered a reliable and safe procedure for investigation of small intestine disorders [10-12]. Incidents of hemorrhagic MD mostly involve young patients; Zheng CF et al. suggest that DBE is a safe and dependable procedure for diagnosis of MD in the pediatric as well as adult population [13]. DBE provides a higher detection rate of MD among the procedures considered (SBCE and CT).

We showed that major and minor findings were the characteristics of hemorrhagic MD and they were not detected in most of the cases of incidental MD. Second, specificity of major findings and sensitivity of the minor finding were high when hemorrhagic and incidental MD were compared. Therefore, 
Table 2 Features of hemorrhagic and incidental MD.

\begin{tabular}{|c|c|c|c|c|}
\hline & $\begin{array}{l}\text { Hemorrhagic MD } \\
(n=25)\end{array}$ & $\begin{array}{l}\text { Incidental MD } \\
(\mathrm{n}=\mathbf{8})\end{array}$ & $P$ value & OR $(95 \% \mathrm{CI})$ \\
\hline Age at initial examination, year, median(range) & $24(9-77)$ & $44(21-78)$ & $P=0.057^{1}$ & \\
\hline Duration of symptoms, days, median(range) & $18(2-4675)$ & $23(4-427)$ & $P=0.38^{1}$ & \\
\hline \multicolumn{5}{|l|}{ Size } \\
\hline Long axis length, cm, median(range) & $6(4-12)$ & $4(2-6)$ & $P=0.031^{1}$ & \\
\hline Short axis length, cm, median(range) & $3(2-8)$ & $2(1-4)$ & $P=0.29^{1}$ & \\
\hline \multicolumn{5}{|l|}{ Finding of double-balloon endoscopy } \\
\hline a. Major findings (ectopic gastric mucosa and/or open ulcer) & 13 & 0 & $P=0.01^{2}$ & $2.1(1.39-3.13)$ \\
\hline i. Ectopic gastric mucosa & 7 & 0 & $P=0.31^{2}$ & \\
\hline ii. open ulcer & 10 & 0 & $P=0.16^{2}$ & \\
\hline \multirow[t]{2}{*}{ b. Minor finding (ring-like scar) } & 22 & 1 & $P<0.01^{2}$ & $51.3(4.58-576.0)$ \\
\hline & Detection rate $(n=23)$ & Number positive & & \\
\hline Mecklel's scan positive) & $40 \%(8 / 20)$ & $0 \%(0 / 3)$ & $P=0.53^{2}$ & \\
\hline $\begin{array}{l}\text { Wireless capsule endoscopy }(n=14) \text { detecting rate } \\
\text { (number of detected MD) }\end{array}$ & $46.1 \%(6 / 13)$ & $0 \%(0 / 1)$ & $P=1^{2}$ & \\
\hline Hb at admisson time, g/dL, median(range) & $7.3(4.2-14.5)$ & $13.3(8.5-14.6)$ & $P<0.01^{2}$ & \\
\hline Blood transfusion volume, $U$, median(range) & $5.2(0-55)$ & 0 & $P=0.018^{2}$ & \\
\hline
\end{tabular}

- Table 3 Relations between Major and Minor findings in Meckel's diverticulum.

\begin{tabular}{|c|c|c|c|c|c|c|c|c|}
\hline & \multicolumn{3}{|c|}{$\begin{array}{l}\text { Hemorrhagic MD (N=25) } \\
\text { Major finding }\end{array}$} & \multicolumn{3}{|c|}{$\begin{array}{l}\text { Incidental MD ( } \mathrm{N}=8) \\
\text { Major finding }\end{array}$} & \multirow[b]{2}{*}{ EGM + ulcer } & \multirow[b]{2}{*}{ None } \\
\hline & $\mathrm{EGM}^{1}$ & Ulcer & EGM + ulcer & None & $\mathrm{EGM}^{1}$ & Ulcer & & \\
\hline \multicolumn{9}{|l|}{ Minor findings } \\
\hline Ring-like scar & 3 & 4 & 4 & 11 & 0 & 0 & 0 & 1 \\
\hline None & 0 & 2 & 0 & 1 & 0 & 0 & 0 & 7 \\
\hline
\end{tabular}

- Table4 Diagnostic yield of endoscopic finding for hemorrhagic MD.

\begin{tabular}{|c|c|c|c|c|c|}
\hline & Accuracy & Sensitivity & Specificity & $\mathbf{P P V}^{1}$ & $\mathbf{N P V}^{2}$ \\
\hline $\begin{array}{l}\text { Major finding (Ectopic gastric mucosa and/or } \\
\text { ulcer) }\end{array}$ & $64 \%(21 / 33)$ & $52 \%(13 / 25)$ & $100 \%(8 / 8)$ & $100 \%(13 / 13)$ & $40 \%(8 / 20)$ \\
\hline a. Ectopic gastric mucosa & $45 \%(15 / 33)$ & $28 \%(7 / 25)$ & $100 \%(8 / 8)$ & $100 \%(/ 7)$ & $31 \%(8 / 26)$ \\
\hline b. ulcer & $55 \%(18 / 33)$ & $40 \%(10 / 25)$ & $100 \%(8 / 8)$ & $100 \%(10 / 10)$ & $35 \%(8 / 23)$ \\
\hline Minor finding (ring-like scar) & $88 \%(29 / 33)$ & $88 \%(22 / 25)$ & $87.5 \%(7 / 8)$ & $96 \%(22 / 23)$ & $70 \%(7 / 10)$ \\
\hline Major and/or minor findings & $94 \%(31 / 33)$ & $96 \%(24 / 25)$ & $87.5 \%(7 / 8)$ & $96 \%(24 / 25)$ & $88 \%(7 / 8)$ \\
\hline
\end{tabular}


the combination of major and minor findings can improve diagnostic confidence in cases of hemorrhagic MD.

Major findings for characterization of MD indicate that it appears to be a promising method. To the best of our knowledge, we have reported for the first time on endoscopic assessment of ectopic gastric mucosa contained in the MD. At EGD, gastric and duodenal mucosa have a completely different surface pattern: gastric mucosa appears flat with a red-colored surface, while duodenal mucosa is characterized by presence of villi and a brighter mucosa. Therefore, when indigo carmine is sprayed during endoscopic procedures, ectopic gastric mucosa (included in the MD) appears reddish, in contrast with the duodenal mucosa ( Fig. 1). In this study, the specificity of endoscopically visualized ectopic gastric mucosa for diagnosing hemorrhagic MD was $100 \%$. This suggests that MD with ectopic gastric mucosa has a high risk of gastrointestinal bleeding. Similarly, the specificity of detecting ulceration in cases of hemorrhagic MD was $100 \%$, suggesting that the ulcer located in the MD sac is an additional pattern of ectopic gastric mucosa.

Although the major findings had high specificity, the sensitivity (52\%) was low. Therefore they cannot be considered pathognomonic. In some cases identification of the ectopic gastric mucosa was challenging due to the limited intra-diverticula area. In other cases, the tightness of the ring-like scar did not allow evaluation of the diverticula.

When endoscopic findings of hemorrhagic and incidental MD were compared, the minor finding had a strong correlation with incidental MD $(P<0.01)$ and was diagnostic of hemorrhagic MD. The sensitivity of major and minor findings is $96 \%$. Therefore, the combination of major and minor findings can drastically improve diagnostic confidence in cases of hemorrhagic MD.

Our study has a few limitations. It is a single-center retrospective study involving a limited number of patients. In addition, 6 patients with hemorrhagic MD included in the study did not have ectopic mucosa. Sonozaki et al. reported that ectopic gastric mucosa was observed in the surgically resected specimen in only 1 of 3 cases with ulcerations [7], suggesting that the cause of the ulcerations in the remaining 2 cases without ectopic gastric mucosa was uncertain. Manneret et al. speculated that mechanical irritation of the tissue included between the ileal lumen and MD was the cause of the ulceration [14]. In our series, we did not confirm these hypotheses and future studies, therefore, are needed.

\section{Conclusion}

In conclusion, hemorrhagic MD can be diagnosed and differentiated from incidental MD based on the combination of major and minor findings at DBE, thus allowing the most appropriate management. In addition, our findings may strengthen diagnosis of incidental MD, avoiding unnecessary surgical procedures [15-17].

\section{Competing interests}

None

References

[1] Weinstein EC, Cain JC, Remine WH. Meckel's diverticulum: 55 years of clinical and surgical experience. JAMA 1962; 182: 251-253

[2] Soltero M], Bill AH. The natural history of Meckel's diverticulum and its relation to incidental removal. Am J Surg 1976; 32: 168-173

[3] Mortensen NJ, Jones O. The small and large intestines. Bailey \& Love's Short Practice of Surgery. 24th ed. 2004: 1159-1160

[4] Pennazio M. Small-bowel capsule endoscopy and device-assisted enteroscopy for diagnosis and treatment of small bowel disorders: European Society of Gastrointestinal Endoscopy (ESGE) Clinical Guideline. Endoscopy 2015; 47: $352-376$

[5] Yamamoto $\mathrm{H}$, Sekine $\mathrm{Y}$, Sato $\mathrm{Y}$ et al. Total enteroscopy with a nonsurgical steerable double-balloon method. Gastrointest Endosc 2001; 53: $216-220$

[6] Mylonaki M, MacLean D, Fritscher-Ravens A et al. Wireless capsule endoscopic detection of Meckel's diverticulum after nondiagnostic surgery. Endoscopy 2002; 34: 1018-1020

[7] Shinozaki S, Yamamoto H, Ohnishi H et al. Endoscopic observation of Meckel's diverticulum by double balloon endoscopy: Report of five cases. J Gastroenterol Hepatol 2008; 23: 308-311

[8] Rutherford RB, Akers DR. Meckel's diverticulum: a review of 148 pediatric patients, with special reference to the pattern of bleeding and tomesodiverticular vascular bands. Surgery 1966; 59: 618-626

[9] Stone PA, Hofeldt M], Campbell JE et al. Meckel diverticulum: ten-year experience in adults. South Med J 2004; 97: 1038-1041

[10] Yamamoto H, Sugano K. A new method of enteroscopy the doubleballoon method. Can J Gastroenterol 2003; 17: $273-274$

[11] Yamamoto H, Yano T, Kita H et al. New system of double-balloon enteroscopy for diagnosis and treatment of small intestinal disorders. Gastroenterology 2003; 125: 1556

[12] Nakamura M, Niwa Y, Ohmiya $\mathrm{N}$ et al. Preliminary comparison of capsule endoscopy and double-balloon enteroscopy in patients with suspected small-bowel bleeding. Endoscopy 2006; 38: 59-66

[13] Zheng CF, Huang Y, Tang ZF et al. Double-balloon enteroscopy for the diagnosis of Meckel's diverticulum in pediatricpatients with obscure GI bleeding. Gastrointest Endosc 2014; 79: $354-358$

[14] Manner H, May A, Nachbar L et al. Push-and-pull enteroscopy using the double-balloon technique (double-balloon enteroscopy) for the diagnosis of Meckel's diverticulum in adult patients with GI bleeding of obscure origin. Am. J. Gastroenterol 2006; 101: 1152-1154

[15] Arnold JF, Pellicane JV. Meckel's diverticulum: a ten-year experience. Am Surg 1997; 63: $354-355$

[16] Rivas H, Cacchione RN, Allen JW. Laparoscopic management of Meckel's diverticulum in adults. Surg Endosc 2003; 17: 620

[17] Chan KW, Lee KH, Wong HY. Laparoscopic excision of Meckel's diverticulum in children: what is the current evidence? World J Gastroenterol 2014; 20: 15158-15162 